\title{
Development of sEMG-based robust oral motion classification method and its application to electric wheelchair operation
}

\author{
Yukiya NAKAI*, Makoto SASAKI*, Katsuhiro KAMATA* and Atsushi NAKAYAMA** \\ ${ }^{*}$ Graduate School of Engineering, Iwate University \\ 4-3-5 Ueda, Morioka, Iwate 020-8551 Japan \\ E-mail: makotosa@iwate-u.ac.jp \\ ** Department of Engineering for Future Innovation, National Institute of Technology, Ichinoseki College \\ Takanashi, Hagisho, Ichinoseki, Iwate 021-8511, Japan
}

Received: 13 March 2019; Revised: 4 September 2019; Accepted: 27 October 2019

\begin{abstract}
Interfaces based on surface electromyography (sEMG) signals are one of the important methods for noninvasively extracting the intention of a severely disabled person and supporting environmental control of wheelchairs and personal computers. However, sEMG-based interfaces generally have a common and maximum disadvantage of vulnerability to changes in electrode position. In this study, we aimed to develop a robust oral motion classification method that is robust to change in electrode position. Five healthy adult male subjects participated in this experiment. sEMG signals of the suprahyoid muscles during five oral motions (right, left, up tongue motion, jaw opening, and clenching) were measured using a boomerang-shaped 22-channel electrode adhered to the underside of the jaw. Oral motion classification from sEMG signals was performed using a support vector machine (SVM). When SEMG signals measured at a position different from the 22-channnel electrode position where the training data for SVM classifier was obtained were used as the test data, the classification accuracy of five oral motions sharply decreased from $92.0 \%$ to $72.8 \%$. In contrast, when the 10 trials of sEMG signals obtained in advance at different electrode positions on different days were used as training data, the robustness against electrode position change was improved drastically and the mean classification accuracy of all subjects reached $90.4 \%$. Furthermore, we developed an electric wheelchair control system that can operate based on classified motions and verified its usefulness for wheelchair operability and driving performance thorough the experiment. The results showed that the proposed method can omit the SVM training process required every time after the electrode is attached and can operate the wheelchair immediately after electrode attachment. Such advancement of interfaces eliminates the annoyance caused to the user who uses the interface on a daily basis and is expected to lead to an improvement in the quality of life.
\end{abstract}

Keywords : Surface electromyography-based interface, Suprahyoid muscles, Oral motion classification, Electric wheelchair, Support vector machine

\section{Introduction}

To support the daily life of severely disabled people, including those with tetraplegia, cervical spine injury, and muscular dystrophy, it is very important to ascertain a person's intention through non-invasively measurable bio-signals; the ascertained intention is used to control the environment, e.g., a wheelchair, personal computer (PC), or smart phone, by extension. Various interfaces focusing on head motion, eye movement, and electroencephalogram (EEG) have been developed (LoPresti et al., 2002; Tanaka et al., 2005; Iturrate et al., 2009; Kim et al., 2015; Eid et al., 2016). The ability of severely disabled people can vary markedly among individuals. In addition, the interfaces that can be used for an individual depend on the presence or absence of progressive disease and physical function deterioration. It is therefore important to develop different types of interfaces and increase user choices to accommodate the various needs of all severely disabled people.

With reference to such backgrounds, we have been developing an interface focusing on tongue motion (Sasaki et al., 
2016). Tongue motor functions are usually preserved even in people with cervical spinal cord damage. Anyone can precisely set their own tongue position and can smoothly change the magnitude of the force imposed on the teeth or palate. Therefore, various tongue interfaces have been proposed so far (Ghovanloo, 2007). For example, an interface using an artificial palate with buttons or pressure sensors that can be activated directly by the tongue tip, and an interface using an artificial palate with a few pairs of light emitting diodes and photodiodes for precisely detecting the tongue position, etc. have been investigated (Clayton et al., 1992; Wrench et al., 1998; Kim et al., 2005; Saponas et al., 2009). An interface using a small permanent magnet attached to the tongue tip and an array of magnetic sensors mounted on a headset outside the mouth was also proposed to detect the tongue position from magnetic field variations inside and around the mouth (Huo et al, 2008; Yousefi et al, 2011; Sahadat et al., 2018). However, these conventional methods require the insertion of the sensors into the mouth cavity directly and additional efforts for maintaining oral hygiene alongside its insertion. They also might entail various difficulties while talking and eating. Therefore, we developed a surface electromyography (sEMG)-based tongue interface to solve these problems (Sasaki et al., 2016). The proposed interface classified tongue motions from sEMG signals of the suprahyoid muscles detected from the underside of the jaw. The sEMG signals of the suprahyoid muscles were measured via a 22-channnel electrode attached on the underside of the jaw. Six tongue motions (right, left, up, down, and forward tongue motion, and tongue motion while swallowing saliva) were classified with an accuracy of 95.1\% from the features of the sEMG signals using a support vector machine (SVM) classifier. Because our approach requires no installation of any sensor in the mouth cavity, the developed interface does not hinder the user's other activities such as eating, chewing, or talking. sEMG signals generally have the disadvantage that the signal component observed differs depending on the electrode mounting position. This is the common and biggest problem of interfaces based on sEMG signals. Therefore, in our system, every time the 22-channnel electrode is attached to the underside of the jaw, it is necessary to associate the sEMG signals with tongue motions using an SVM (i.e., SVM training process). This training process is cumbersome for users who routinely use the interface, and it is strongly required to improve and advance the interface so that the training process can be skipped.

In this study, by using the multiple sEMG signals obtained in advance at different 22-channnel electrode positions on different days as training data of SVM, we developed a robust oral motion classification method that can omit the SVM training process required every time after the electrode is attached to the underside of the jaw. The developed method was applied for classification of not only tongue motions but also jaw motions. Furthermore, we developed an electric wheelchair control system that can operate based on these oral motions classified by the proposed method and verified its usefulness thorough experiments.

\section{Tongue interface that uses sEMG signals of suprahyoid muscles}

In this study, based on the tongue motion classification method developed by the authors in a previous study (Sasaki et al., 2016), we realize a robust oral motion classification method that is robust to the 22-channnel electrode position change. Oral motions include both tongue and jaw motions.

The tongue motion classification method comprises the sEMG measurement, feature extraction, and motion classification.

\section{1 sEMG signal measurement}

The target muscles of sEMG signal measurement are suprahyoid muscles. Suprahyoid muscles are composed of four muscles (digastric muscle, stylohyoid muscle, mylohyoid muscle, and geniohyoid muscle), and they are involved in any oral motion such tongue motion, lower jaw motion, or swallowing (Fig. 1).

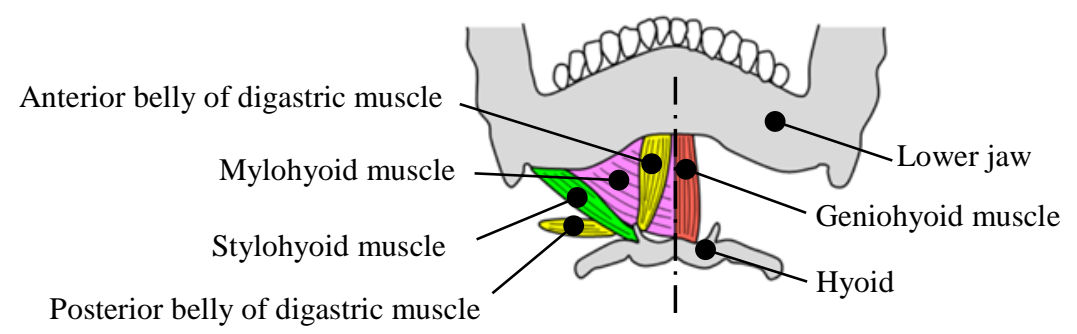

Fig. 1 Structure of suprahyoid muscles. 
A 22-channel electrode designed as a thin flexible boomerang-shaped patch was adhered to the underside of the jaw of the subject (Fig. 2) to observe the muscle activity of the entire suprahyoid muscles. A ground electrode and an indifferent electrode were also connected to the left and right earlobes of the subject, respectively, via ear clips. The electric potential between each electrode of the 22-channel electrode and the indifferent electrode was amplified using a separate differential amplifier with reference to the potential of the ground electrode. The gain of the differential amplifier was set at 2,052. A band-pass filter with a passband from 16 to $440 \mathrm{~Hz}$ bandwidth was used to remove the noise superimposed on sEMG signals. sEMG signals of all 22 sEMG channels were digitized using a 16-bit analog-to-digital converter (USB-6218; National Instruments Corp.). The sampling rate was 2,000 Hz.
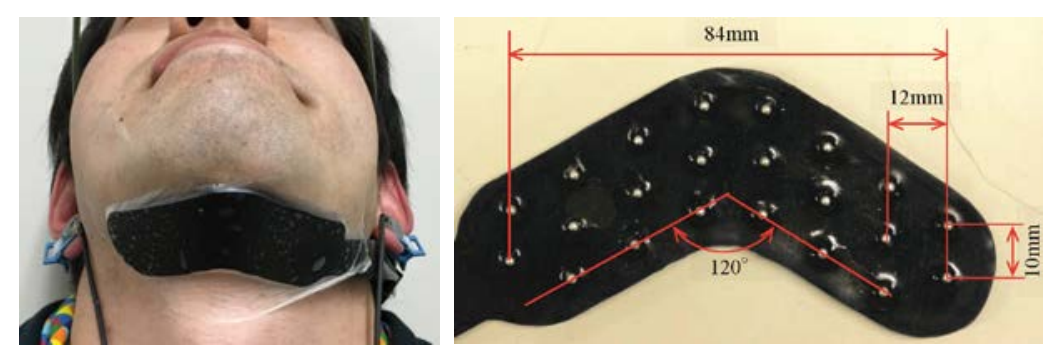

Fig. 2 The 22-channel electrode.

\subsection{Feature extraction}

For feature extraction, sEMG signals measured from all 22 channels were segmented into data windows comprising 256 samples (128 ms). The next sampling segment was slid over the current one after a time increment of 16 ms (Englehart et al., 2001). As a feature, the root mean square (RMS) and the cepstrum coefficients (CC) of the sEMG signals were calculated for each window and each channel (Zecca et al., 2002; Yoshikawa et al., 2007). The RMS features are characteristics of a time domain and provide amplitude information of sEMG signals. The CC features are characteristics of the frequency domain, and low-order CC include information about the spectral envelope of the power spectrum of sEMG signals. For the tongue motion classification, low-order (1-5) CC were selected as CC features. The dimension of the feature vector is 132 ((1-dimensional RMS features + 5-dimensional CC features) $\times 22$ channels).

\subsection{Motion classification}

The SVM classifier was used for the motion classification. Training data comprising pairs of feature vectors and correct class labels are required for training SVM. Correct class labels that correspond to the feature vectors can be obtained by threshold triggering of sEMG signals. Then, using training data, a decision function for classifying each motion was created using an SVM classifier with a radial basis function kernel. The hyperparameters (kernel parameter $\gamma$ and penalty parameter C) of the SVM were optimized using a grid search process within training data.

After training the SVM, motion classification was performed using unknown sEMG signals (i.e., test data). Predicted class labels were replaced with a "neutral” tongue position when all sEMG signals were under the threshold level (i.e., relaxed state). Then, to reduce the effect of possible misclassification, a majority-voting technique was applied to a moving window comprising 10 frames, and the final predicted class label with the largest number of wins was obtained. Finally, the classification accuracy to the test data was calculated using the following equation:

Classification accuracy $=\frac{\text { number of correctly predicted class labels }}{\text { total number of predicted class labels }} \times 100(\%)$.

\section{Development of the robust oral motion classification method}

The common issue of interfaces using sEMG signals is the observed signal change due to the electrode position change. In our interface, it is impossible to mount the 22 channel electrode at the same position every time. Since the relative position of the electrode and muscles is necessarily different, the measured sEMG signals of the suprahyoid muscles are changed. Therefore, every time the 22-channnel electrode is attached to the underside of the jaw, it is necessary to associate the sEMG signals with oral motions using an SVM (i.e., SVM training process). When using the sEMG-based interface on a daily basis, it is troublesome for the user to perform the training process every time; therefore, 
it is essential to develop an oral motion classification method that is robust to electrode position change. In this study, we aimed to develop an interface that is robust to electrode position change.

\subsection{Experimental protocol}

Five healthy adult male subjects $(22.6 \pm 1.5$ years old, $174.4 \pm 5.3 \mathrm{~cm}$ tall, $56.2 \pm 4.5 \mathrm{~kg}$ weight, mean \pm SD) participated in this experiment. The oral motion set included three tongue motions (right, left, and up tongue motion performed with closed mouth) and two jaw motions (jaw opening and clenching) (Fig. 3). During tongue motions, subjects were asked to position their tongue tips sequentially in the maxillary right second molar tooth, the maxillary left second molar tooth, and the hard palate. In the experiment, each oral motion was executed for 4 s. A resting period of 4 $\mathrm{s}$ was allowed before the start of the next motion. Consequently, all five oral motions in one set were completed for a total of 36 s. These five oral motions were performed as 1 set, and 7 sets of sEMG data per 1 trial were measured.

Further, sEMG signal measurement for 1 trial was performed on another day to obtain the sEMG dataset measured at a different electrode position. We repeated this measurement for 20 days and obtained a total of 20 trials of sEMG data (i.e., sEMG signals of five oral motions $\times 7$ sets $\times 20$ trials). Incidentally, the precise attachment position of the 22 channel electrode to the underside of the jaw was not stipulated and the subjects attached it to the position at which they felt comfortable.

Approval for tests was obtained in advance by the Ethical Review Board of Iwate University. Before the start of tests, the study objectives, experimental protocol, and risks were explained to each subject, and written consent was received from all participants.

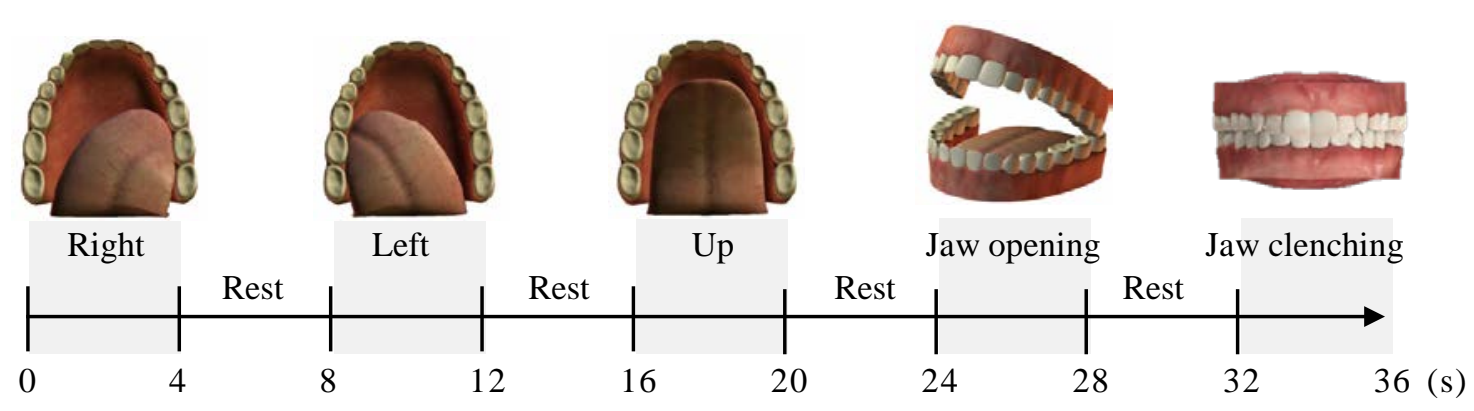

Fig. 3 Illustration of the five oral motions in the set.

\subsection{Verification of classification accuracy at same electrode positions}

In this section, we verify the classification accuracy of five oral motions when the 22-channel electrode position is the same.

\subsubsection{Analysis method}

Classification accuracy of the oral motions was calculated for each trial. The first 2 sets of the 7 total sEMG measurement sets were used as training data for SVM classifier and the remaining 5 sets were used as test data for the evaluation of classification accuracy. Finally, the mean classification accuracy for a total of 20 trials was calculated.

In training process, the search range of hyperparameters of SVM was set to $\gamma=\left\{2^{-3}, 2^{-2}, \ldots, 2^{4}\right\}$ and $C=\left\{2^{2}, 2^{3}, \ldots\right.$, $\left.2^{7}\right\}$. The optimal combination with the highest classification rate was defined using fivefold cross validation. The LIBSVM library was used for SVM training and oral motion classification (Hsu et al., 2016).

\subsubsection{Results}

Figure 4 shows the classification results for all five subjects. The lowest value of mean classification accuracy was $90.8 \%$ for subject D and the highest was $93.9 \%$ for subject $\mathrm{E}$. The average for all subjects was $92.0 \%$. Therefore, it was shown that if the 22-channnel electrode position does not change, the oral motions of all subjects can be classified with an accuracy of more than $90 \%$. 


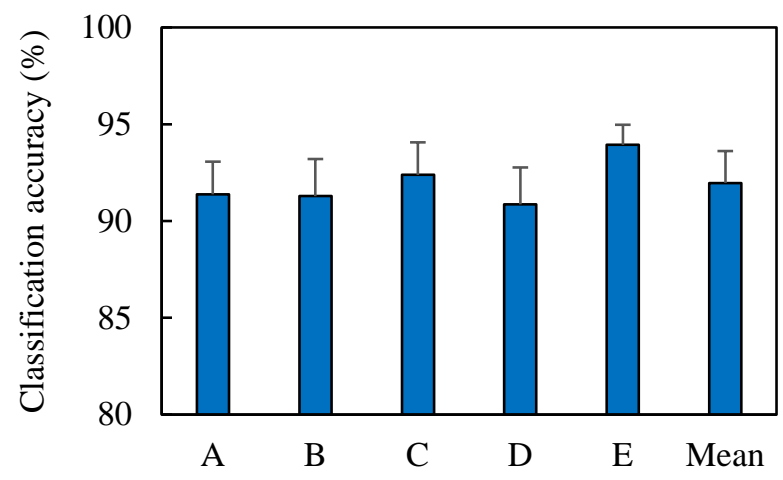

Fig. 4 Classification accuracy of oral motions at same electrode positions.

\subsection{Verification of classification accuracy at different electrode positions}

In this section, we confirm the classification accuracy of five oral motions when the 22-channel electrode position changes and develop an oral motion classification method that is robust to electrode position change.

\subsubsection{Analysis method}

In this study, by using the multiple sEMG signals obtained in advance at different 22-channnel electrode positions on different days as training data of SVM, we realize an oral motion classification method that is robust to electrode position change. Based on this concept, the sEMG signals of the 1st and 2nd sets in the 1st to 10th trials (i.e., total 20 sets $=2$ sets $\times 10$ trials) were used as training data for the SVM classifier (Fig. 5). The sEMG signals of the 3rd to 7th sets in the 11th to 20 th trials (i.e., total 50 sets $=5$ sets $\times 10$ trials) were used as test data for the evaluation of classification accuracy.

To evaluate the influence of the amount of training data obtained from different 22-channel electrode positions on the classification accuracy, changes in classification accuracy when the training data was increased one by one from 1 to 10 trials were calculated.

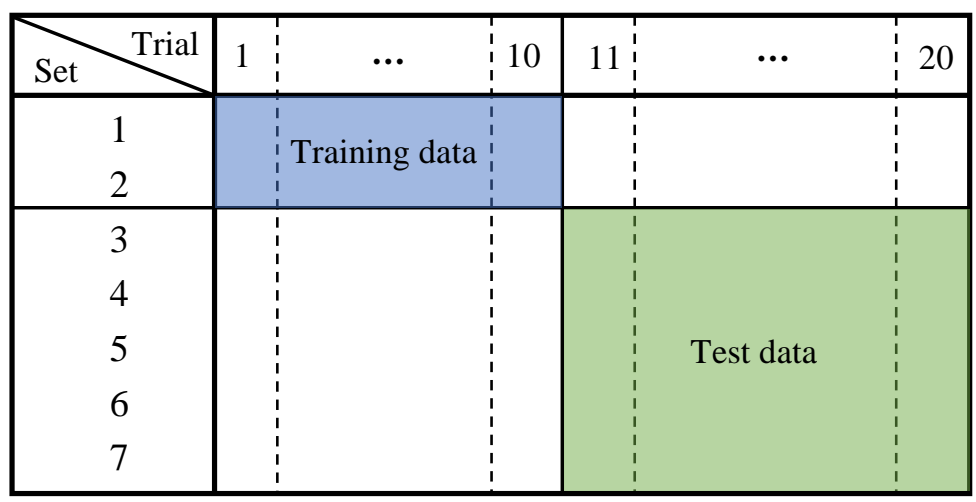

Fig. 5 Define of training and test data.

\subsubsection{Results}

Figure 6 shows the relation of the amount of training data and the classification accuracy of five oral motions. When the amount of training data was 1 trial, the classification accuracy to the test data obtained at different 22-channel electrode positions was low.

The lowest classification accuracy was $35.8 \%$ for subject $\mathrm{B}$, the highest was $86.6 \%$ for subject $\mathrm{D}$, and the mean for all subjects was $72.8 \%$. In contrast, the classification accuracy improved when the amount of training data was increased. When the amount of training data was 10 trials, the lowest classification accuracy was $88.7 \%$ for subject $\mathrm{D}$ and the highest was $91.9 \%$ for subject $\mathrm{E}$. The mean classification accuracy for all subjects improved significantly from $72.8 \%$ to $90.4 \%$. 


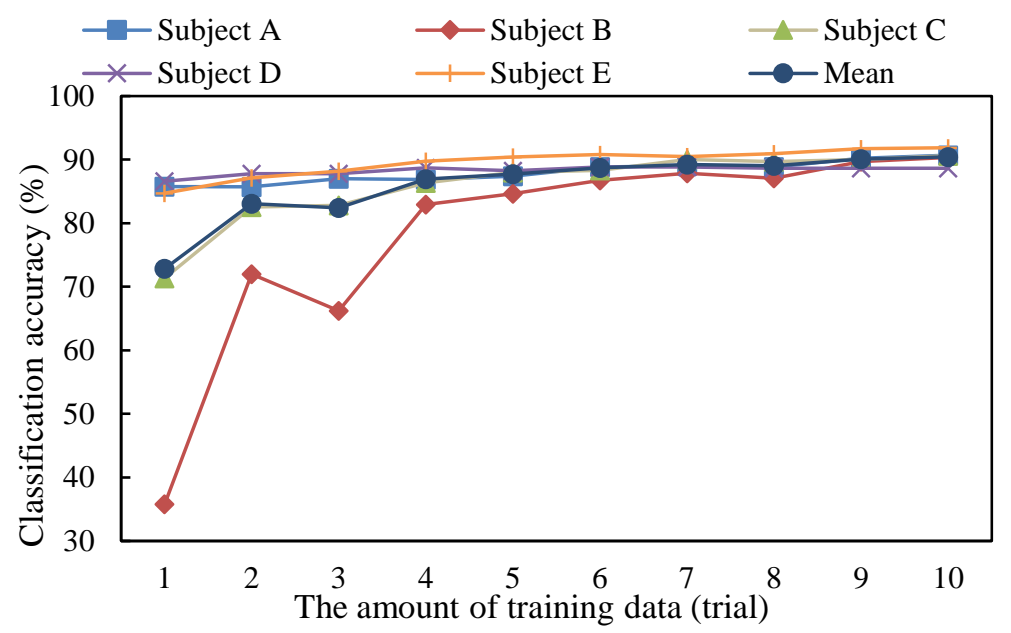

Fig. 6 Relation of the amount of training data and the classification accuracy.

\subsection{Discussion}

The sEMG data for total 20 trials used in this study are data sets that can classify five oral motions with $>90 \%$ accuracy in any subject unless the electrode position changes (i.e., remains the same for the training and test data; Fig. 4). However, when sEMG signals measured at different positions were used as the test data, the classification accuracy decreased sharply; the lowest classification accuracy was $35.8 \%$ and the mean for all subjects was $72.8 \%$ (Fig. 6). This vulnerability to the electrode position change is a common and the biggest disadvantage of an interface using sEMG signals. In contrast, when sEMG data of 10 trials measured at different electrode positions were used as the training data, the robustness against electrode position change improved drastically and the mean classification accuracy of all subjects reached $90.4 \%$. This classification accuracy nearly equals to the accuracy when the electrode position is same (92.0\%), confirming the usefulness of our proposed method.

Using a 22-channel electrode can be a reason for the increase in the robustness to the electrode position. In general, it is impossible to attach the electrode every time at the same position on the skin surface. However, the 22-channel electrode used in this study has the following advantages.

i) The 22-channel electrode was designed as a thin flexible boomerang-shaped patch to cover the entire jaw and to observe the muscle activity of the entire suprahyoid muscles.

ii) For the same subject, the relative position of each electrode constituting the 22-channel electrode hardly changes depending on the place (i.e., underside of the jaw) where the electrode is attached.

Therefore, by repeatedly attaching and detaching the 22-channnel electrode about ten times and collecting the sEMG signals at each electrode position in advance, it is thought that SVM could consider not only the difference in muscle activity pattern depending on the type of oral motion but also the difference in muscle activity patterns depending on the electrode position, as individual characteristics for oral motion classification. These results suggest that the robust oral motion classification method proposed in this study can omit the SVM training process required every time after the 22channel electrode is attached to the underside of the jaw. Furthermore, even if the electrode position shifts while using this interface, there is a small influence on the classification accuracy, still improving the interface's safety and reliability. From the above, it can be said that this study realized a user-friendly sEMG-based interface that is robust to electrode position change. This finding is expected to serve as a clue in how to solve common problems of many sEMG-based interfaces.

\section{Development of an electric wheelchair operated using oral motions}

In the previous section, we proposed the oral motion classification method that lends robustness to electrode position change. Here, we develop an electric wheelchair control system that can operate using the proposed method and verify its usefulness via experiments. 


\subsection{Electric wheelchair}

In this study, an electric wheelchair (EMC-920, Imasen Engineering Corp.) that can be operated freely (as with a joystick operation) by inputting two independent analog voltages to the motor controller was used. The control command for the wheelchair operation was generated based on classified oral motions and was transmitted via Bluetooth wireless communication. The PC used to generate and transmit the control command was the same as the PC used for sEMG measurement and oral motion classification. The transmitted control command was received by the Bluetooth module (RN-42, Microchip Technology Inc.) and then transmitted to the AVR microcomputer (Arduino Due, Arduino) by serial communication. Based on the received command, the AVR microcomputer outputs a 2-channel analog voltage for operation of the electric wheelchair and inputs it to the motor controller. With such a series of flows, the electric wheelchair is controlled. In this way, by using Bluetooth communication, the electric wheelchair and the driver are electrically insulated and the safety for the human body has been secured. In addition, noise suppression given to sEMG measurement was realized at the same time. In this experiment, for further safety, a manual switch for emergency stopping was also installed in the electric wheelchair.

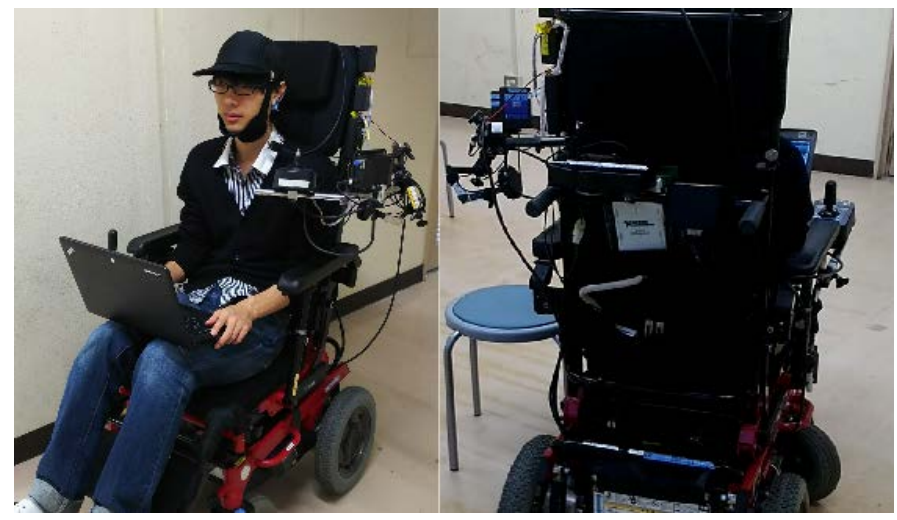

Fig. 7 Appearance of the electric wheelchair used for experiment.

\subsection{Control method}

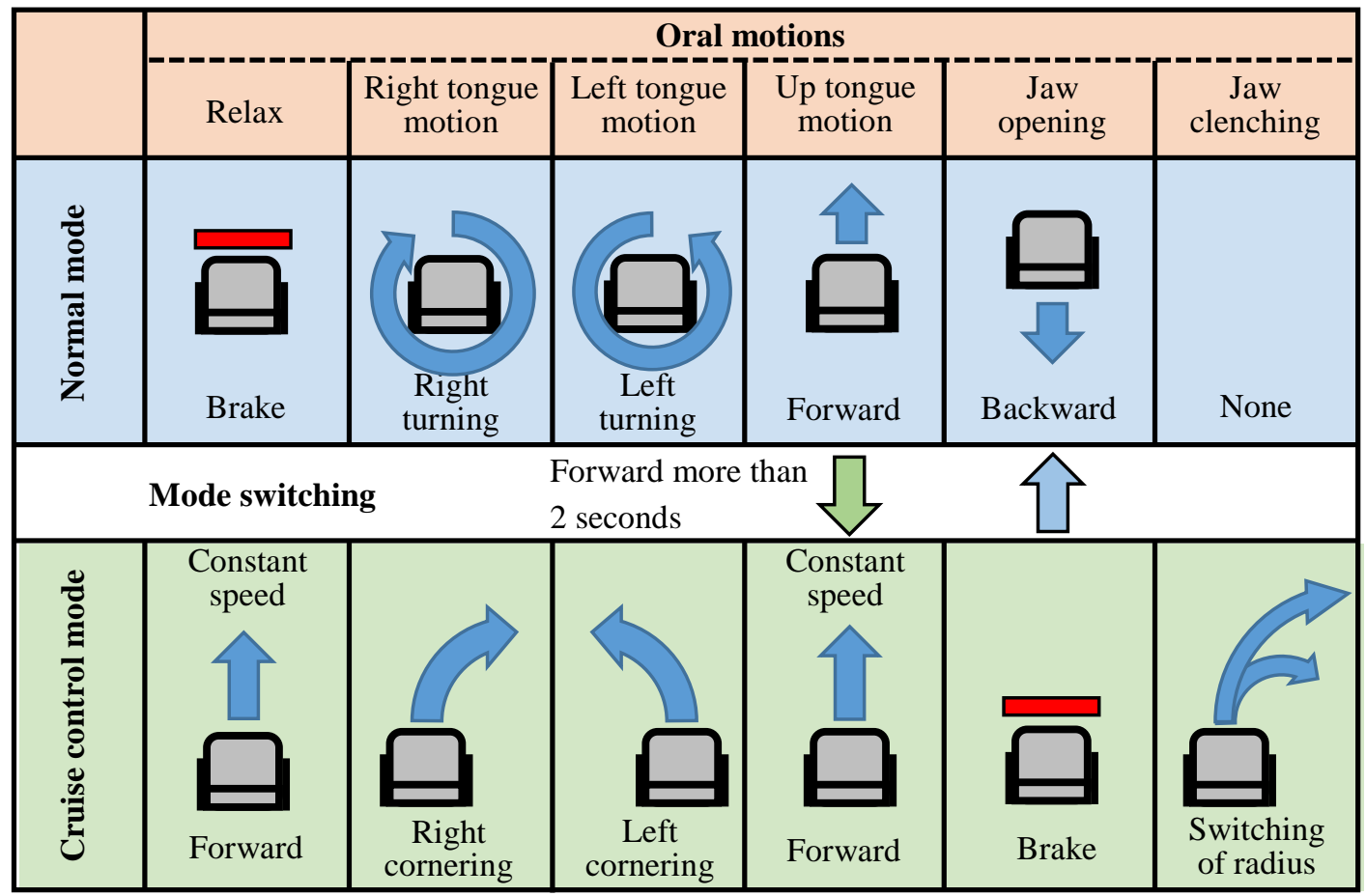

Fig. 8 Correspondence between oral motions and wheelchair behaviors in each mode. 
For wheelchair operation using oral motions, two modes were provided (Fig. 8). In the normal mode, the brake command is sent to the wheelchair when all sEMG signals are under the threshold level (i.e., relaxed state). The right and left tongue motions were linked with the right and left turning commands, respectively. The up tongue motion and jaw opening motion were linked with the forward and backward commands, respectively. If the forward command is sent to the wheelchair for more than 2 seconds, the mode switches from normal mode to cruise control mode. Cruise control mode aims to reduce tongue fatigue by enabling the wheelchair to continue moving forward at a constant speed despite the relaxed tongue state. In this mode, right and left tongue motions were used as commands for fine adjustment of the traveling direction, respectively. These tongue motions were also linked with the right and left cornering commands, respectively. The radius of the cornering can switch between $0.6 \mathrm{~m}$ and $5 \mathrm{~m}$ at any time by jaw clenching. The jaw opening was used as the brake command and simultaneously switches mode from cruise control to normal. When the mode is changed, a notification sound is generated by the PC to easily communicate the current mode to the driver.

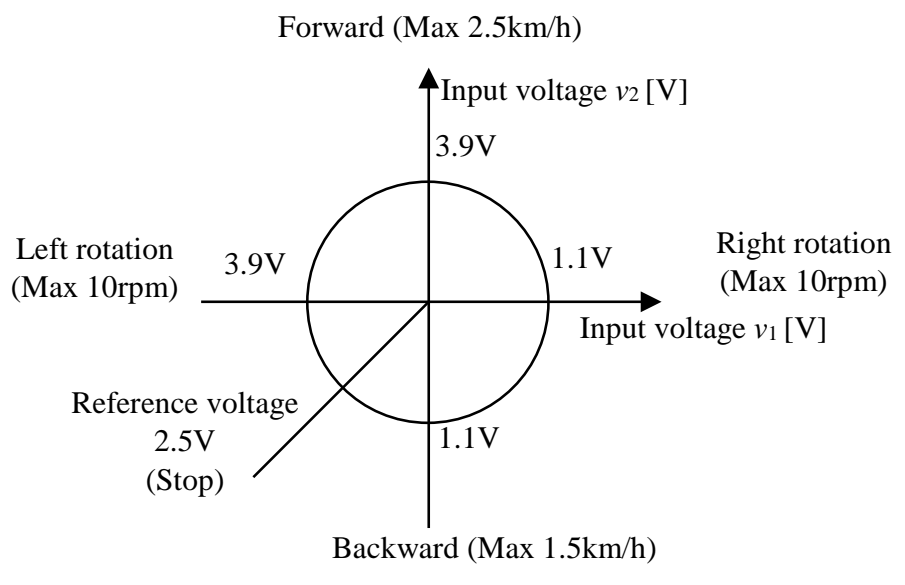

Fig. 9 Relation between wheelchair behavior and input voltages.

Figure 9 shows the correspondence between the electric wheelchair behavior and the input voltages to the motor controller. The oral motions classified from sEMG signals were converted into input voltages $v_{1}$ and $v_{2}$ corresponding to the electric wheelchair operations. Both the range of input voltages $v_{1}$ and $v_{2}$ were set from $1.1 \mathrm{~V}$ to $3.9 \mathrm{~V}$. The reference voltage was $2.5 \mathrm{~V}$. For example, the inputs of $v_{1}=2.5 \mathrm{~V}$ and $v_{2}=3.9 \mathrm{~V}$ indicate moving forward with maximum speed, and the inputs of $v_{1}=2.5 \mathrm{~V}$ and $v_{2}=1.1 \mathrm{~V}$ indicate moving backward with maximum speed. However, if such voltages corresponding to the maximum speed are input to the motor controller during stoppage or other operation, there is a risk of causing sudden acceleration/deceleration, sudden turning, and wheelchair swaying. Similar risks arise if drivers undertake unintentional oral motion or when the classification accuracy of oral motion from sEMG signals is $100 \%$ or less. Therefore, in this research, the input voltages $v_{1}$ and $v_{2}$ were determined as follows to solve these problems.

$$
\begin{aligned}
& v_{1}(i)=v_{r e f}+\frac{v_{r}}{(a / F)} S_{1}(i), \\
& v_{2}(i)=v_{r e f}+\frac{v_{r}}{(a / F)} S_{2}(i),
\end{aligned}
$$

where $v_{r e f}$ is the reference voltage $(2.5 \mathrm{~V}), v_{r}$ is variable voltage range $(1.4 \mathrm{~V}=3.9-2.5 \mathrm{~V}=2.5-1.1 \mathrm{~V})$, $a$ is acceleration time to reach maximum speed ( $1 \mathrm{~s}), F$ is update cycle of input voltage (64 ms), $i$ is number of update times $(i=1,2, \ldots$, $t / F)$, and $t$ is operating time of wheelchair. $S_{1}$ and $S_{2}$ are variables that are updated according to the classification result of the oral motions.

$$
\begin{aligned}
& S_{1}(i)=S_{1}(i-1)+C_{1}(i), \\
& S_{2}(i)=S_{2}(i-1)+C_{2}(i),
\end{aligned}
$$

where $C_{1}$ and $C_{2}$ are given in Table 1 . The minimum value of $C_{1}$ and $C_{2}$ is $-a / F$ and the maximum value is $a / F$. 
Table 1 Definition of $C_{1}, C_{2}$.

\begin{tabular}{c|cc}
\hline Classified oral motion & $C_{1}(i)$ & $C_{2}(i)$ \\
\hline Relax & $N_{1}(i)$ & $N_{2}(i)$ \\
Right tongue motion & +1 & $N_{2}(i)$ \\
Left tongue motion & -1 & $N_{2}(i)$ \\
Up tongue motion & $N_{1}(i)$ & +1 \\
Jaw opening & $N_{1}(i)$ & -1 \\
\hline
\end{tabular}

$N_{1}$ and $N_{2}$ in Table 1 are values that stably bring the input voltages $v_{1}$ and $v_{2}$ closer to the voltage corresponding to the target operation of the wheelchair, and these determined below in (6) and (7).

$$
\begin{aligned}
& N_{1}(i)= \begin{cases}+1 & \left(S_{1}(i-1)<0\right) \\
+0 & \left(S_{1}(i-1)=0\right) \\
-1 & \left(S_{1}(i-1)>0\right)\end{cases} \\
& N_{2}(i)= \begin{cases}+1 & \left(S_{2}(i-1)<0\right) \\
+0 & \left(S_{2}(i-1)=0\right) \\
-1 & \left(S_{2}(i-1)>0\right)\end{cases}
\end{aligned}
$$

With this controlling method, since the input voltages $v_{1}$ and $v_{2}$ increase/decrease smoothly, rapid acceleration/deceleration of the electric wheelchair can be avoided. In addition, since the influence of incorrect classification of oral motions is suppressed, even if oral motions cannot be classified with $100 \%$ accuracy, it is expected that a driver's intended driving can be provided.

\subsection{Experiment}

Each of the five subjects described in Section 3 also participated in this experiment. Here, we sought to verify the effectiveness of the proposed robust oral motion classification method for the electric wheelchair operation. The test course set on a flat road surface indoors was $1.2 \mathrm{~m}$ wide and the total distance was about $23 \mathrm{~m}$ (Fig. 10). Each subject was given 10 min of advance practice to learn how to operate the wheelchair in each mode. To stop the wheelchair's forward motion in cruise control mode and switch from cruise control to normal operating mode, the subjects were instructed to perform the jaw opening motion once and after that the relaxed state could be maintained. Then, the following two methods, a) and b), were alternately conducted five times for the electric wheelchair driving experiments. To perform the wheelchair driving experiments at different 22-channnel electrode positions, the electrode was reattached for each driving test. That is, the total number of times of desorption of the electrode was 10 times.

a) Conventional method: A method wherein 2 sets of training data are obtained immediately after attachment of a 22-channnel electrode to the underside of the jaw and then SVM learning is performed using the data. Oral motion classification and electric wheelchair operation are performed at the same position as the electrode position where the training data was obtained.

b) Proposed method: A method wherein SVM training is performed in advance using the all sEMG signals of the 1st and 2 nd sets in the 1 st to 10 th trials (i.e., total 20 sets $=2$ sets $\times 10$ trials) described in Fig. 5 as training data. Oral motion classification and electric wheelchair operation are performed at electrode positions different from the electrode positions where the training data was obtained. Naturally, the day on which oral motion classification and electric wheelchair operation are performed is different from the day on which training data were obtained.

For the evaluation of driving experiments, driving time and sensory evaluation were used. The driving time was defined as the time until the electric wheelchair started driving from the start line and returned to the start line. As a sensory evaluation, a questionnaire composed of 9 items concerning the operability and driving performance of the electric wheelchairs was conducted after driving. The items were as follows: (1) ease of straight driving, (2) ease of turning, (3) ease of cornering, (4) ease of braking, (5) accuracy of wheelchair behavior against oral motion, (6) time lag of wheelchair behavior against oral motions, (7) comfort of driving speed, (8) comfort of riding, and (9) fatigue feeling of the tongue. The organoleptic evaluation was a five-point evaluation, the standard was 3 points, and the highest evaluation point was 5 . 


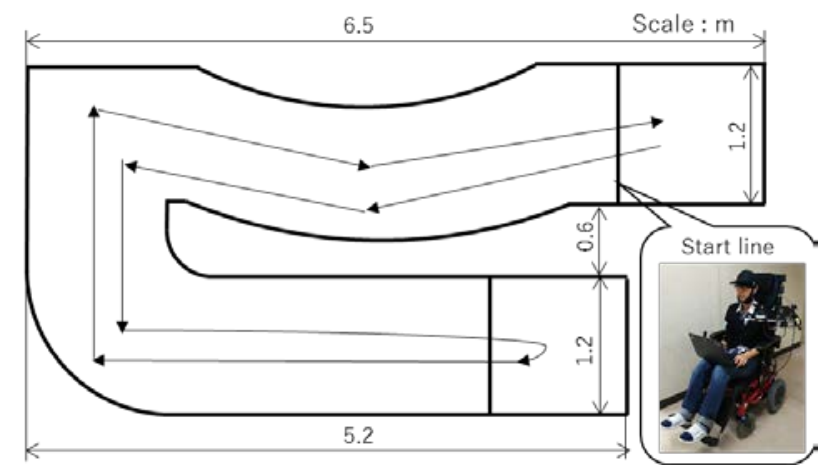

Fig. 10 Test course of electric wheelchair driving experiments.

\subsection{Result}

Table 2 shows the average driving time of each subjects. The average driving time for the conventional method and the proposed method was $154.4 \mathrm{~s}$ and $157.5 \mathrm{~s}$, respectively. There was no statistically significant difference in these driving times as a result of a $t$-test. However, the SD of driving times for the proposed method was smaller than SD for the conventional method for all subjects.

Table 2 Average driving time.

\begin{tabular}{c|cc|cc}
\hline & \multicolumn{2}{|c|}{ Conventional method } & \multicolumn{2}{c}{ Proposed method } \\
& Time [s] & SD & Time [s] & SD \\
\hline Subject A & 169.6 & 47.6 & 206.9 & 36.3 \\
Subject B & 164.0 & 54.6 & 156.0 & 14.6 \\
Subject C & 172.3 & 77.6 & 194.2 & 41.1 \\
Subject D & 131.3 & 37.1 & 120.2 & 21.0 \\
Subject E & 136.7 & 54.5 & 110.2 & 14.8 \\
Mean & 154.4 & 54.9 & 157.5 & 25.6 \\
\hline
\end{tabular}

口Conventional method $\quad$ aProposed method

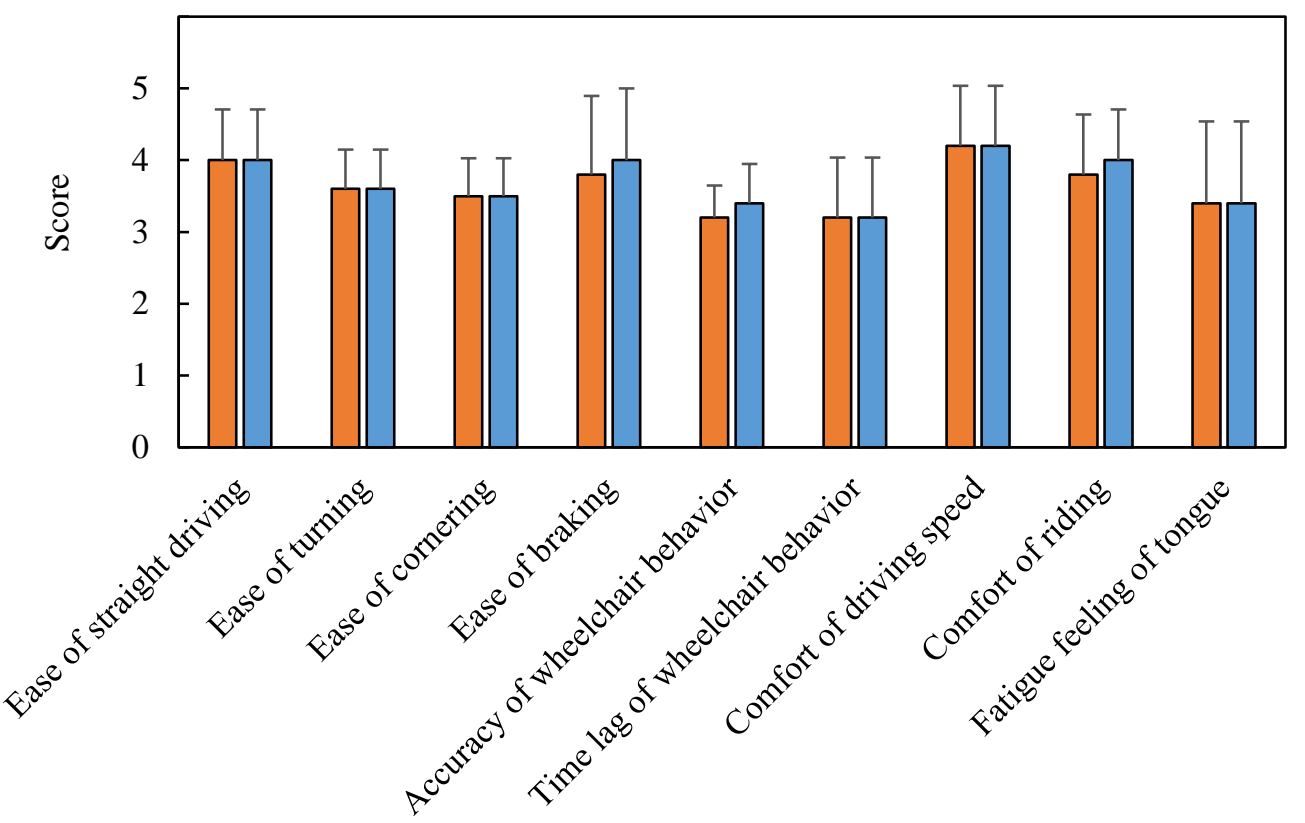

Fig. 11 Average score of sensory evaluation. 
Figure 11 shows the result of the sensory evaluation. The evaluation score of "(6) time lag of wheelchair behavior against oral motions” received the lowest score of 3.2 for both the conventional and proposed methods. In addition, “(5) accuracy of wheelchair behavior against oral motion” earned a similarly low score. In contrast, items “(1) ease of straight driving” and “(7) comfort of driving speed” received high scores of more than 4 points in both methods. Additionally, in any of these 9 items, there were no statistical differences between both methods. Therefore, it was confirmed that by using the proposed method, wheelchair operability and driving performance equivalent to those of the conventional method could be obtained.

\subsection{Discussion}

In general, the sEMG signal components observed differs depending on the electrode mounting position. Therefore, to realize stable oral motion classification, it is ideal to measure training data immediately after mounting the 22-channnel electrode and associate the relationship between the sEMG signals and the oral motions by SVM. This is the approach of the conventional method shown in Section 4.3. However, experimental results for wheelchair driving clarified that wheelchair driving time, wheelchair operability, and driving performance equivalent to those of the conventional method can be obtained using the proposed classification method, which is robust to electrode position change. This suggests that omitting the training process of SVM, which was indispensable after mounting of the 22-channnel electrode, has no influence on the operation of the electric wheelchair. The training process is troublesome for users who use the interface on a daily basis, and having solved this point, it could prove to be very useful and epoch-making.

In Table 2, it is shown that the SD of driving time for the proposed method was smaller than that for the conventional method for all subjects. The head movement for checking the course causes a slight change in the relative position of the muscle and the electrode and the observed sEMG signal components change so that the classification accuracy of the oral motions during wheelchair driving may temporarily be reduced. In the proposed method, since the muscle activity pattern measured at different electrode positions in advance is included in the training data, it is expected that the influence was hardly affected by such a change in the sEMG signal components. Therefore, the effectiveness of the proposed method was suggested also from the viewpoint of stability against electric wheelchair operation.

From Fig. 11, it is shown that the sensory evaluation score of "(6) time lag of wheelchair behavior against oral motions" obtained the lowest score. There are two possible reasons why subjects felt a time lag. The first is that the majority-voting technique was applied to the output signal of the SVM. The majority-voting technique can reduce the influence of the unexpected misclassification of oral motions, while the time shift (delay) of the signal usually increases with increasing number of votes. The second is that the decision algorithm of the input voltages $v_{1}$ and $v_{2}$ for the motor control was devised and applied to reduce the risk of wheelchair's sudden acceleration/deceleration and sudden turning. In our controlling method, since the input voltages $v_{1}$ and $v_{2}$ increase/decrease smoothly, thereby allowing time lag to occur slightly, but influence of misclassification of oral motion on wheelchair operation can be reduced. To support the mobility of a severely disabled person who does not have sufficient upper limb function for joystick operation, it is effective to more reliably realize the operation based on one's own will rather than realizing the same level of operability as the joystick operation. Therefore, in this research, we focused on the safety of wheelchair operation, i.e., suppression of the bad influence of misclassification of oral motions rather than the response speed of the wheelchair to oral motions. In the future, it will be necessary to investigate the relationship between allowable time lag and operability and clarify control parameters for wheelchair operation suitable for each severely disabled person.

However, the oral motion classification method proposed in this study includes approximately $10 \%$ misclassification. The behavioral result of such misclassification is the electric wheelchair trying to move in a direction different from that intended by the subjects. In this experiment, the electric wheelchair never did not lose control or run away because of the implementation of both the majority-voting technique and the decision algorithm of the input voltages $v_{1}$ and $v_{2}$. However, the momentary undesirable behavior of the electric wheelchair led to anxiety for the subjects, thus likely resulting in a low score in the category of "(5) accuracy of wheelchair behavior against oral motion.” This anxiety may increase for the subjects when driving in a narrow aisle or near a pedestrian. Therefore, to reduce this anxiety of the subjects, further improvement of the accuracy of oral motion classification is necessary. Moreover, further improvement of the overall safety of wheelchair driving is important, and this can be achieved by combining accurate oral motion classification with external sensors such as those used for obstacle avoidance and environment recognition. 


\section{Conclusion}

In this study, we aimed to develop an oral motion classification method that is robust to electrode position change. When sEMG signals measured at a position different from the 22-channnel electrode position where the training data for SVM classifier was obtained were used as the test data, the classification accuracy of five oral motions (right, left, and up tongue motion, jaw opening, and clenching) sharply decreased from $92.0 \%$ to $72.8 \%$. This vulnerability to the electrode position change is the most common and biggest disadvantage of interfaces using sEMG signals. In contrast, when the 10 trials of sEMG signals obtained in advance at different electrode positions on different days were used as training data, the robustness against electrode position change was improved drastically and the mean classification accuracy of all subjects reached $90.4 \%$. Furthermore, we developed an electric wheelchair control system that can operate based on these oral motions as classified by our proposed method and verified its usefulness for wheelchair operability and driving performance via experiments. Results showed that the proposed method can omit the training process required every time after the 22-channel electrode is attached to the underside of the jaw and can operate the electric wheelchair immediately after attachment of an electrode. Such advancement of interface eliminates the annoyance caused to the user who uses the interface on a daily basis and is expected to lead to an improvement in their quality of life. In the future, we will further investigate the safety of the electric wheelchair operation using the proposed interface and it will be necessary to verify its usefulness for severely disabled people.

\section{Acknowledgement}

This study was supported in part by the Grant-in-Aid for Scientific Research (B), Japan Society of Promotion of Science (Principal Investigator: Makoto Sasaki, Grant Number: 18H03557).

\section{References}

Clayton, C., Platts, R.G.S., Steinberg, M. and Hennequin, J.R. Palatal tongue controller, Journal of Microcomputer Applications, Vol.15 (1992), pp.9-12.

Eid, M.A., Giakoumidis, N. and Saddik, A.E., A novel eye-gaze-controlled wheelchair system for navigating unknown environments: case study with a person with ALS, IEEE Access, Vol.4 (2016), pp.558-873.

Englehart, K., Hudgins, B. and Parker, P.A., A wavelet-based continuous classification scheme for multifunction myoelectric control, IEEE Transactions on Biomedical Engineering, Vol.48, No.3 (2001), pp.302-311.

Ghovanloo, M., Tongue operated assistive technologies. Proceedings of the IEEE 29th engineering medicine biology conference (2007), pp.4376-4379.

Hsu, C.W., Chang, C.C. and Lin, C.J., A Practical guide to support vector classification (2016), https://www.csie.ntu.edu.tw/ cjlin/ (accessed 2019-2-28)

Huo, X., Wang, J. and Ghovanloo, M., Introduction and preliminary evaluation of the tongue drive system: wireless tongue-operated assistive technology for people with little or no upper-limb function, Journal of Rehabilitation Research \& Development, Vol.45, No.6 (2008), pp.921-930.

Iturrate, I., Antelis, J.M., Kubler, A. and Minguez, J., A noninvasive brain-actuated wheelchair based on a P300 neurophysiological protocol and automated navigation, IEEE Transactions on Robotics, Vol.25 (2009), pp.614-627.

Kim, D., Tyler, M.E. and Beebe, D.J., Development of a tongue operated switch array as an alternative input device, International Journal of Human-Computer Interaction, Vol.18 (2005), pp.19-38.

Kim, M., Kim, B.H. and Jo, S., Quantitative evaluation of a low-cost noninvasive hybrid interface based on EEG and eye movement, IEEE Transactions on Neural Systems and Rehabilitation Engineering, Vol.23 (2015), pp.159-168.

LoPresti, E.F., Brienza, D.M. and Angelo, J., Head-operated computer controls: effect of control method on performance for subjects with and without disability, Interacting with Computers, Vol.14 (2002), pp.359-377.

Sahadat, M.N., Dighe, S., Islam, F. and Ghovanloo, M., An independent tongue-operated assistive system for both access and mobility, IEEE Sensors Journal, Vol.18, No.22 (2018), pp. 9401-9409.

Saponas, T.S., Kelly, D., Parviz, B.A. and Tan, D.S., Optically sensing tongue gestures for computer input, Proceedings of the 22nd annual ACM symposium on user interface software and technology (2009), pp 177-180.

Sasaki, M., Onishi, K., Stefanov, D., Kamata, K., Nakayama, A., Yoshikawa, M. and Obinata, G., Tongue interface based on surface EMG signals of suprahyoid muscles, ROBOMECH Journal, Vol.3, No.9 (2016), pp.1-11.

Tanaka, K., Matsunaga, K. and Wang, H.O. Electroencephalogram-based control of an electric wheelchair, IEEE Transactions on Robotics, Vol.21 (2005), pp.762-766. 
Wrench, A., McIntosh, A.D., Watson, C. and Hardcastle, W.J., Optopalatograph: real-time feedback of tongue movement in 3D, Proceedings of the fifth international conference on spoken language processing (1998), pp.1867-1870.

Yoshikawa, M., Mikawa, M. and Tanaka, K., A myoelectric interface for robotic hand control using support vector machine, Proceedings of the 2007 IEEE/RSJ international conference on intelligent robots and systems (2007), pp.2723-2728.

Yousefi, B., Huo, X., Kim, L., Veledar, E. and Ghovanloo, M., Quantitative and comparative assessment of learning in a tongue-operated computer input device: navigation tasks, IEEE Transactions on Information Technology in Biomedicine, Vol.15, No.5 (2011), pp.747-757.

Zecca, M., Micera, S., Carrozza, M.C. and Dario, P., Control of multifunctional prosthetic hands by processing the electromyographic signal, Critical Reviews in Biomedical Engineering, Vol.30 (2002), pp.459-485. 